\title{
The burden of chronic obstructive pulmonary disease among employed adults
}

This article was published in the following Dove Press journal:

International Journal of COPD

16 March 2012

Number of times this article has been viewed

\section{Marco daCosta \\ DiBonaventura' \\ Ryne Paulose-Ram² \\ Jun $\mathrm{Su}^{3}$ \\ Margaret McDonald ${ }^{2}$ \\ Kelly H Zou ${ }^{2}$ \\ Jan-Samuel Wagner ${ }^{1}$ \\ Hemal Shah ${ }^{3}$}

'Health Sciences Practice, Kantar Health, New York, NY, USA; '2Pfizer, Inc, New York, NY, USA; ${ }^{3}$ Boehringer Ingelheim Pharmaceuticals, Inc, Ridgefield, CT, USA
Correspondence: Marco DiBonaventura Health Sciences Practice, Kantar Health, II Madison Avenue, 12th Floor,

New York, NY 10010 , USA

Tel + I 2127063988

Fax +I 2126477659

Email marco.dibonaventura@

kantarhealth.com
Objective: To examine quality of life, work productivity, and health care resource use among employed adults ages 40-64 years with chronic obstructive pulmonary disease (COPD) in the United States.

Methods: Data from the 2009 National Health and Wellness Survey were used. All employed adults ages 40-64 years with or without a self-reported diagnosis of COPD were included in the study. Impact on quality of life (using the mental and physical component summary scores and health utilities from the Short Form-12v2), work productivity and activity impairment (using the Work Productivity and Activity Impairment questionnaire), and resource use were analyzed using regression modeling.

Results: There were 1112 employed adults with COPD versus 18,912 employed adults without COPD. After adjusting for demographics and patient characteristics, adults with COPD reported significantly lower mean levels of mental component summary (46.8 vs 48.5), physical component summary (45.6 vs 49.2 ), and health utilities ( 0.71 vs 0.75 ) than adults without COPD. Workers with COPD reported significantly greater presenteeism (18.9\% vs $14.3 \%)$, overall work impairment (20.5\% vs $16.3 \%)$, and impairment in daily activities (23.5\% vs $17.9 \%)$ than adults without COPD. Employed adults with COPD also reported more mean emergency room visits ( $0.21 \mathrm{vs}$ 0.12 ) and more mean hospitalizations $(0.10$ vs 0.06$)$ in the previous 6 months than employed adults without COPD. All of the above differences were significant at two-sided $P<0.05$.

Conclusion: After adjusting for various confounders, employed adults with COPD reported significantly lower quality of life and work productivity, and increased health care resource utilization than employed adults without COPD. These results highlight the substantial impact and burden of COPD in the United States workforce.

Keywords: chronic obstructive pulmonary disease, quality of life, work productivity, activities of daily living, health care resource use

\section{Introduction}

Chronic obstructive pulmonary disease (COPD) is the fourth leading cause of death in the United States. ${ }^{1}$ Although it has been diagnosed in more than 10 million people, as many as 24 million may have airflow limitation consistent with COPD. ${ }^{1}$ Historically, measures of pulmonary function, such as the forced expiratory volume in 1 second test, have been the standard markers for physiological change in COPD, but have only been weakly associated with dyspnea (shortness of breath) and other symptoms. ${ }^{2}$ Thus, patient-reported outcomes such as health-related quality of life (HRQoL) and work productivity have become important measures in COPD therapy. ${ }^{3}$

In an effort to improve COPD management, much research has focused on the complex interrelationships between the HRQoL of COPD patients and the determining 
factors thereof. Age, gender, disease severity, smoking, socioeconomic status, and psychological factors have been studied in detail, even if little agreement about their effects on HRQoL has been made. ${ }^{4-8}$ However, few studies have examined the impact of COPD among the US workforce. Previous studies have shown that the diagnosis of COPD was associated with a reduction in workforce participation and disability. ${ }^{9,10}$ Among those patients who are able to maintain their place in the workforce, however, the extent of COPD burden is unclear. While some studies have investigated the impact of the disease on absenteeism (time missed from work), research on the subtle effects of presenteeism (lost productivity while at work) have largely been neglected. ${ }^{11}$

Although resource use has been documented among younger employees diagnosed with $\mathrm{COPD},{ }^{12}$ studies typically did not assess self-reported health care utilization. An approach from the employee perspective could complement this literature, especially when comparing resource use of COPD workers to controls. The aim of this study is to assess the impact of COPD on HRQoL, work productivity, and resource use among employed adults.

\section{Methods}

\section{Sample}

Data were obtained from 75,000 respondents who completed the 2009 US National Health and Wellness Survey (NHWS), an annual, cross-sectional study of patients aged 18 years or older. The self-administered, Internet-based questionnaire was given to a sample population identified through a web-based consumer panel whose members were recruited through opt-in emails, co-registration with panel partners, e-newsletter campaigns, online banner placements, and both internal and external affiliate networks. All panelists explicitly agreed to become panel members, registered through unique email addresses, and completed in-depth demographic registration profiles. A stratified random sampling procedure was implemented, using strata based on gender, age, and race/ethnicity, in order for the sample to be representative of the demographic composition of the general population. The study was approved by the Essex Institutional Review Board.

Of the 501,239 who were contacted, 92,759 responded (an 18.5\% response rate). Of those who responded, 75,000 gave their informed consent, met the inclusion criteria (aged 18 years or over), and completed the survey instrument. The demographic composition of the US NHWS sample is comparable to that of the US adult population as assessed by the Current Population Survey of the US Census Bureau and prevalence estimates of various conditions from NHWS are consistent with other well-established sources such as the National Health Interview Survey. ${ }^{13}$ In the current study, data were analyzed only for workers (full-time employed, part-time employed, or self-employed) aged 40-64 years $(n=20,024)$.

\section{Measures COPD diagnosis}

All NHWS respondents were asked a series of questions regarding conditions they might have previously experienced. Those who self-reported experiencing chronic bronchitis, emphysema, or COPD in the past were then asked whether their condition had been diagnosed by a physician. Those who self-reported a diagnosis of COPD, who were 40-64 years of age and employed (full-time, part-time, or self-employed) were compared with those not diagnosed with COPD, who were 40-64 years of age and employed (see Figure 1). No other information (clinical or otherwise) was used to define the groups.

\section{Demographics}

Data on each worker's age, gender, race/ethnicity (nonHispanic white, non-Hispanic black/African-American, Hispanic, or other), highest educational level attained (college degree or more vs less than college degree), previous year's household income $(<\$ 25,000, \$ 25,000$ to $<\$ 50,000, \$ 50,000$ to $<\$ 75,000, \$ 75,000$ or more, or decline to answer), health insurance (yes vs no), health insurance with prescription coverage (yes vs no), and type of employment (full-time, part-time, or self-employed) were assessed.

\section{Health history}

Workers also provided data on their body mass index (BMI) level (categorized by reported weight and height: underweight $(<18.5)$, normal (18.5-24.9), overweight (25.0-29.9), obese ( $\geq 30)$, and missing BMI information, smoking status (current smoker, former smoker, or never smoker), exercise behavior (exercised in the past month vs not exercised in the past month), and alcohol use (current drinker vs non-drinker). Self-reported diagnosis of asthma and the estimation of the Charlson comorbidity index (CCI) were also measured. The CCI captures the extent of comorbid burden and is calculated by weighting the presence of the following conditions and summing the result: HIV/AIDS, metastatic tumor, lymphoma, leukemia, any tumor, moderate/ severe renal disease, hemiplegia, diabetes, mild liver disease, ulcer disease, connective tissue disease, chronic pulmonary 


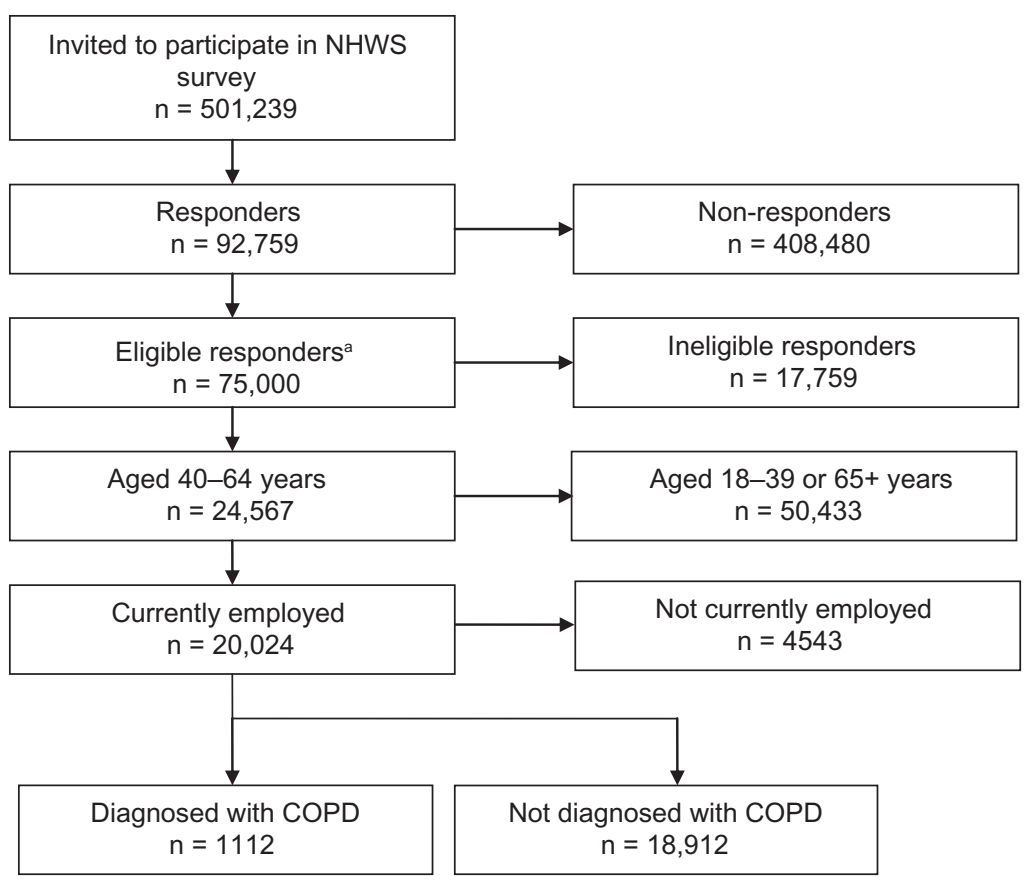

Figure I Inclusion and exclusion flow chart.

Note: aEligible responders included those who provided informed consent, were aged 18 years and over, and completed the survey instrument.

Abbreviations: NHWS, National Health and Wellness Survey; COPD, chronic obstructive pulmonary disease.

disease, dementia, cerebrovascular disease, peripheral vascular disease, myocardial infarction, and congestive heart failure (note that the adjusted CCI does not include diabetes with end organ damage and moderate/severe liver disease which appears in the original CCI; otherwise the calculations are identical to the original algorithm).

\section{HRQoL}

HRQoL was assessed using the SF-12v2, a generic HRQoL instrument which includes twelve items. ${ }^{14}$ The SF-12v2 instrument has an extensive body of literature to support its validity and reliability. ${ }^{14}$ The mental component summary and physical component summary scores were used in the current study. These summary scores are normed to the US population and have a mean of 50 and a standard deviation of 10 . Health state utilities, preference-based single index measures of health, were also calculated from the SF-12 items, and included as the SF-6D. Utility scores vary from 0 (death) to 1 (full health).

\section{Work productivity and activity impairment}

Work productivity and impairment were assessed using the Work Productivity and Activity Impairment Questionnaire: General Health. ${ }^{15}$ Derived from the questionnaire were four metrics: absenteeism (the percentage of work time missed due to health in the past 7 days), presenteeism (the percentage of impairment while at work due to health in the past 7 days), overall work loss (the total percentage of missed time due to absenteeism and presenteeism in the past 7 days), and activity impairment (the percentage of impairment suffered during daily activities in the past 7 days). Each metric varies from $0 \%$ to $100 \%$ with higher scores indicating greater impairment.

Absenteeism is calculated by dividing the number of work hours a worker missed in the past week because of their health by the total number of hours they could have worked (the number of hours they did work plus the number of hours they missed because of their health), then converting this proportion into a percentage. Presenteeism was measured by a worker's reported level of impairment experienced while at work in the past 7 days (from 0 to 10), which was then multiplied by ten to create a percentage. Overall work impairment was measured by combining absenteeism and presenteeism. Activity impairment was measured by a worker's response to their level of impairment experienced in daily activities in the past 7 days (from 0 to 10), which was then multiplied by ten to create a percentage.

The Work Productivity and Activity Impairment Questionnaire: General Health has been found to have adequate reliability and validity. ${ }^{15}$ The scale has also been used in a variety of disease areas including asthma ${ }^{16}$ and has been used to measure differences in those with and without particular diseases to assess burden of illness. ${ }^{13,17,18}$ 


\section{Health care resource use}

Health care utilization was defined by traditional health care providers such as general practitioners and internists ("which of the following traditional health care providers have you seen in the past 6 months?"). Additionally, the number of traditional health care visits, the number of emergency room (ER) visits ("how many times have you been to the ER for your own medical condition in the past 6 months?"), and the number of times hospitalized in the past 6 months ("how many times have you been hospitalized for your own medical condition in the past 6 months?") were included in the analyses.

\section{Analyses}

Univariate analyses were conducted on all workers in order to fully describe the sample demographically. Weights (based on 2008 March Current Population Survey) were then applied to the sample to project to the US population. Using the chi-square and $t$-tests for categorical and continuous variables, respectively, and Wilcoxon-Mann-Whitney tests for continuous non-normal variables differences between those diagnosed with COPD, employed, aged 40-64 years and those not diagnosed with COPD, employed, aged 40-64 years were examined on demographic and health history variables.

Multivariable regression analyses were performed to determine whether the COPD group differed from the control group on HRQoL, work productivity, and resource use after adjusting for demographic (reference categories: male, full-time employed, white, single, college educated, income of less than $\$ 25,000$, no health insurance) and health history variables (reference categories: not diagnosed with asthma, normal weight, never smoked). Our statistical approach varied depending upon the nature of the dependent variable. Multiple regressions were used for HRQoL variables since the SF-12v2 is normed, and generalized linear models (specifying a negative binomial distribution and a log-link function) were used for work productivity and resource utilization, to adjust for nonnormality in the Work Productivity and Activity Impairment Questionnaire: General Health scores and resource use variables. The estimates based on the generalized linear models (work productivity and resource utilization) represent changes in adjusted log values in the given outcome, rather than adjusted values in the outcome, itself. Logistic regressions were used to predict the presence or absence of traditional and non-traditional health care provider visits. All analyses were conducted using SAS (v 9.1; SAS Institute Inc, Cary, NC) and a two-tailed threshold of $P<0.05$ was specified to determine statistical significance.

\section{Results \\ Univariate analyses}

A total of 20,024 workers were employed and aged 40-64 years. Of those, 1112 were diagnosed with COPD and 18,912 were not diagnosed with COPD. Among those employed, most were male (53.4\%), non-Hispanic white $(70.3 \%)$, married or living with a partner (65.7\%), and had received a college education $(81.3 \%)$. Most workers were employed full-time (70.3\%).

\section{Unadjusted comparisons}

Workers with COPD were significantly less likely to be male (45.54\% vs 53.89\%, $P<0.0001$ ), non-Hispanic black/ African-American (8.75\% vs $11.57 \%, P=0.0012)$, Hispanic (8.52\% vs $12.24 \%, P=0.0008)$, full-time employed $(64.65 \%$ vs $70.60 \%, P<0.0001$ ), of normal weight $(21.05 \%$ vs $25.59 \%$, $P=0.0005)$, overweight ( $30.10 \%$ vs $35.91 \%, P=0.0004)$, to have missing BMI information ( $1.21 \%$ vs $1.95 \%, P=0.0458)$, and to have a household income of $\$ 75,000$ or more per year (26.59\% vs $34.42 \%, P<0.0001)$.

Workers with COPD were significantly more likely to be non-Hispanic white $(76.45 \%$ vs $69.96 \%, P<0.0001)$ or other race/ethnicity ( $5.04 \%$ vs $2.62 \%, P=0.0103)$, part-time employed (16.13\% vs $13.13 \%, P=0.0091)$, self-employed (19.22\% vs $16.27 \%, P=0.0174)$, diagnosed with asthma (28.00\% vs $6.23 \%, P<0.0001)$, obese (46.01\% vs $36.18 \%$, $P<0.0001)$, have a household income of less than $\$ 25,000$ per year $(13.55 \%$ vs $10.00 \%, P=0.001)$, and have a household income of $\$ 25,000$ to $\$ 49,999$ per year $(33.20 \%$ vs $27.16 \%, P<0.0001)$. COPD workers reported significantly worse health outcomes than non-COPD workers as captured by HRQoL, work productivity, and health care resource use (see Table 1).

\section{Multivariable analyses with adjustments}

After adjusting for demographic and health history variables, a number of outcome measures were significantly associated with COPD diagnosis. Workers with COPD had significantly lower levels of mental component summary (adjusted means: $\mathrm{COPD}=46.76$ vs control $=48.51$, $P<0.0001$ ), physical component summary (adjusted means: $\mathrm{COPD}=45.64$ vs control $=49.23, P<0.0001$ ), and health utility scores (adjusted means: $C O P D=0.71$ vs control $=0.75, P<0.0001)($ see Figure 2$)$. 


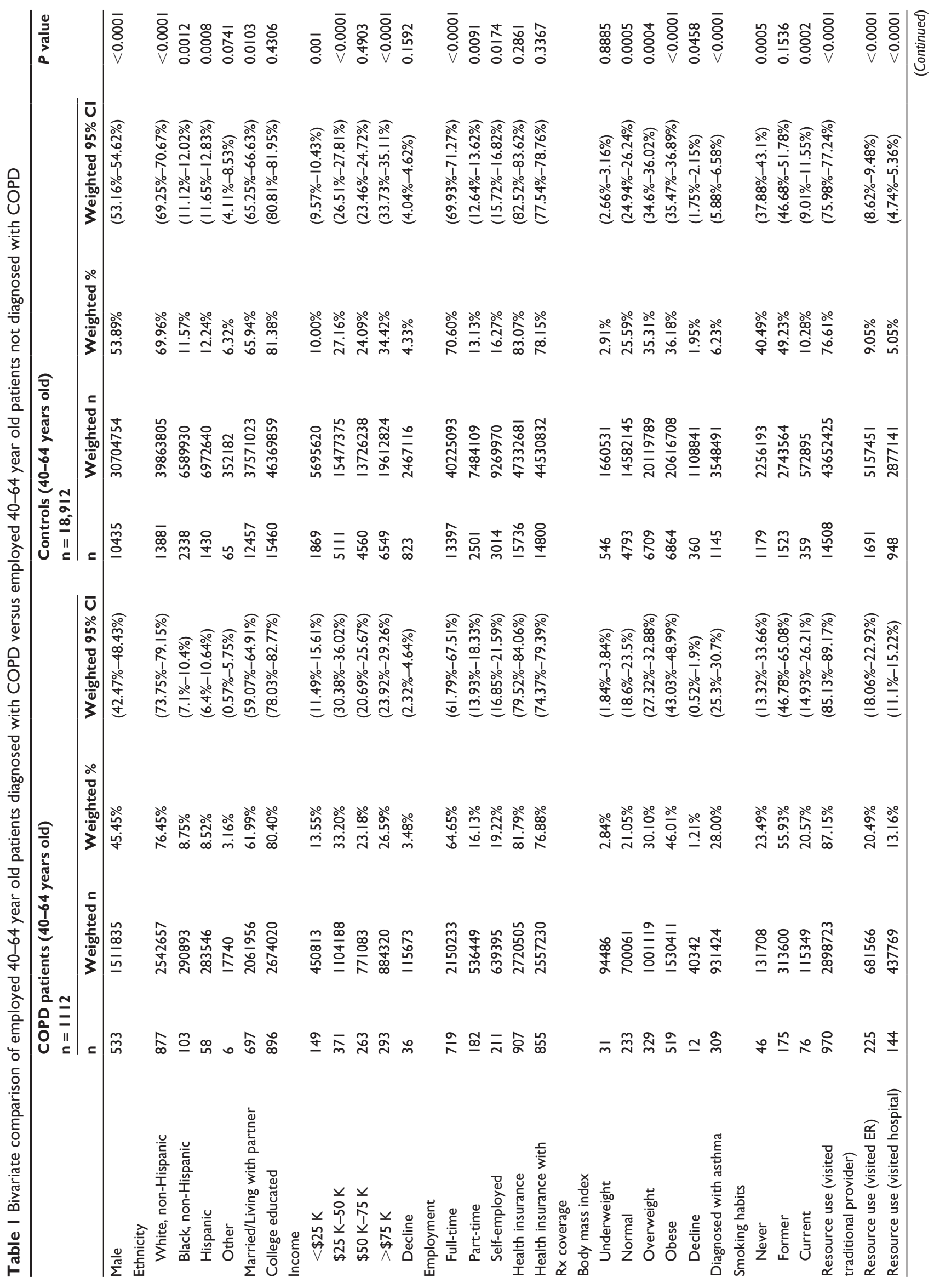




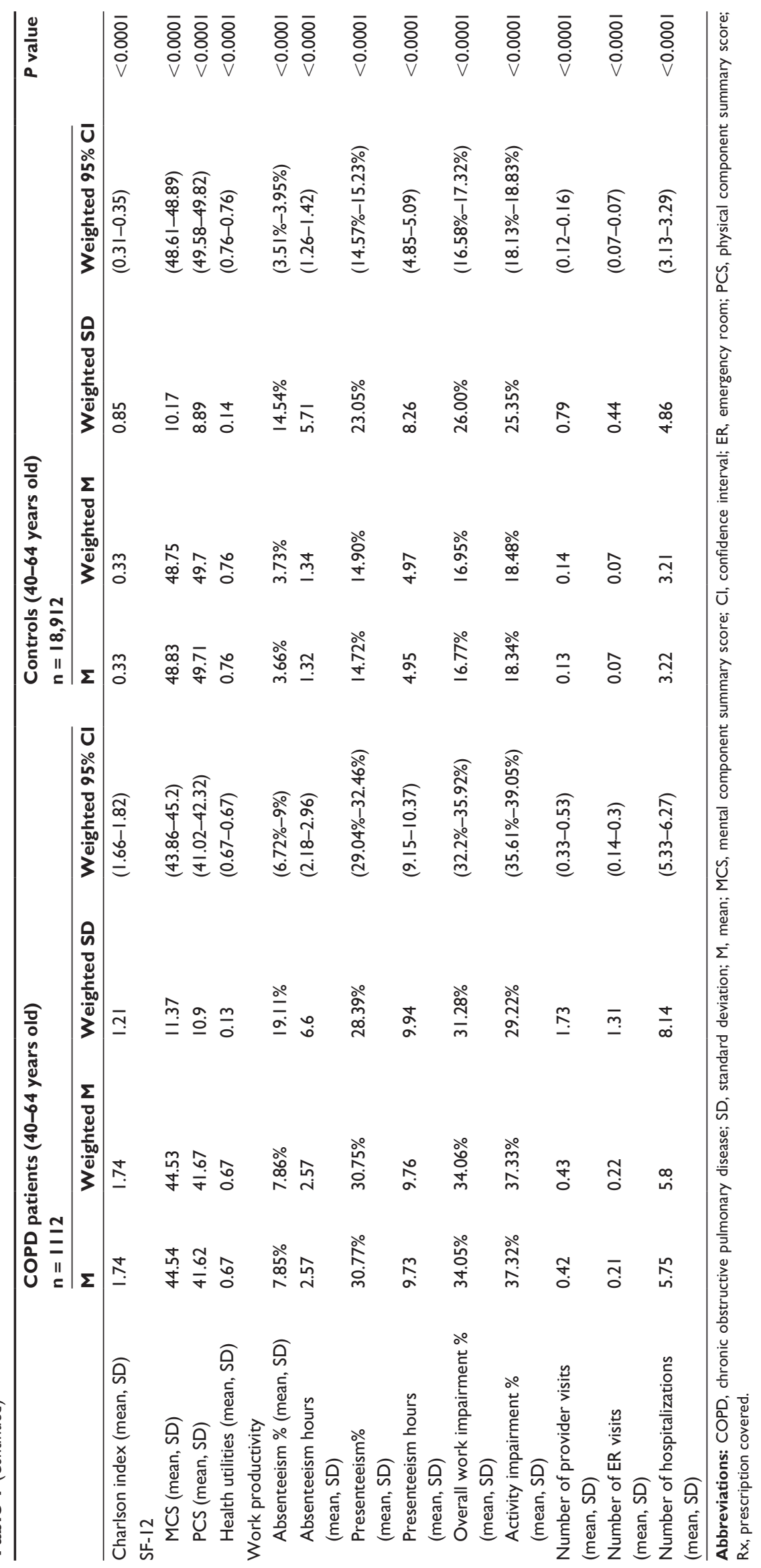




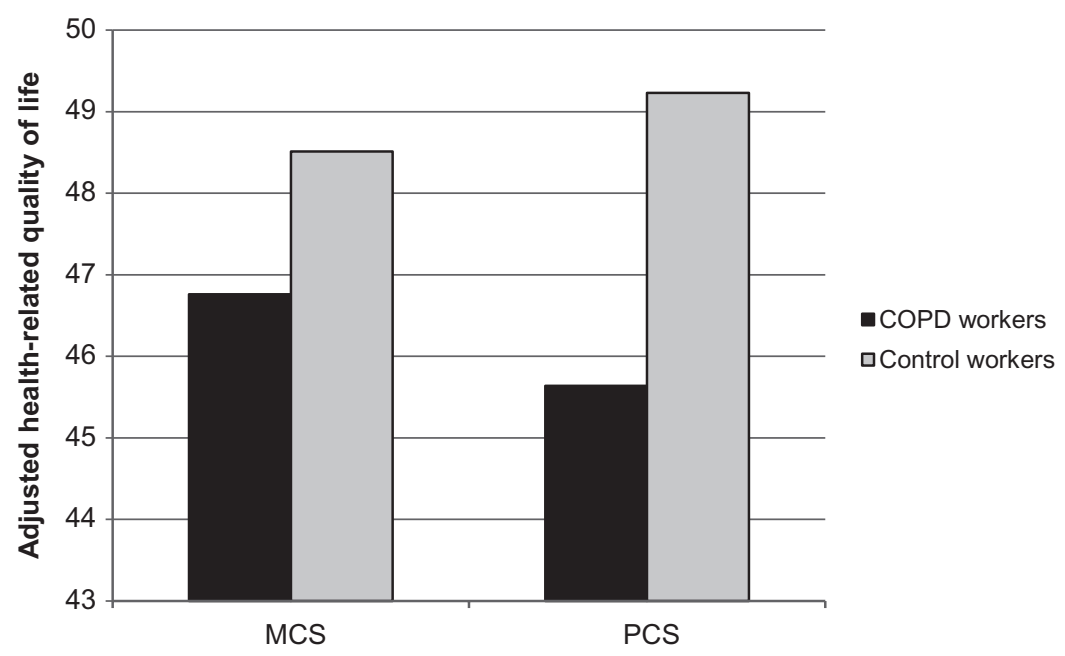

Figure 2 Mean levels of quality of life components after adjusting for patient demographic and patient characteristics (comparing workers with COPD versus those without). Note: Differences in MCS and PCS were found to be statistically significant $(P<0.000 \mathrm{I})$.

Abbreviations: COPD, chronic obstructive pulmonary disease; MCS, mental component summary score; PCS, physical component summary score.

Although there were no significant differences between the groups in regard to absenteeism (adjusted means: $\mathrm{COPD}=3.60 \%$ vs control $=3.42 \%, P=0.7180$ ), workers with COPD reported significantly higher levels of presenteeism (adjusted means: $\mathrm{COPD}=19.28 \%$ vs control $=14.59 \%$, $P<0.0001$; see Figure 3). The composite overall work loss metric was also significantly different between the cohorts (adjusted means: $\mathrm{COPD}=20.93 \%$ vs control $=16.59 \%$ ). Activity impairment, or lost productivity outside of work, was also significantly higher among those who reported a COPD diagnosis (adjusted means: $\mathrm{COPD}=23.95 \%$ vs control $=18.23 \%, P<0.0001)$.

COPD workers reported a significantly higher number of ER visits in the past 6 months (adjusted means:
$\mathrm{COPD}=0.22$ vs control $=0.12, P<0.0001)$, hospitalizations (adjusted means: $\mathrm{COPD}=0.11$ vs control $=0.06$, $P<0.0001$ ) in the past 6 months (see Figure 4), odds of visiting a traditional health care provider (odds ratio $=0.69$, $P=0.0008)$, and odds of visiting a non-traditional health care provider $(\mathrm{b}=1.167, P=0.0401)$. However, no significant differences were found in the number of traditional health care provider visits (adjusted means: $\mathrm{COPD}=3.33$ vs control $=3.06$ )

\section{Discussion}

Although previous studies have examined the impact of COPD on patient-reported outcomes, few studies have focused exclusively on the workforce. Further, prior research

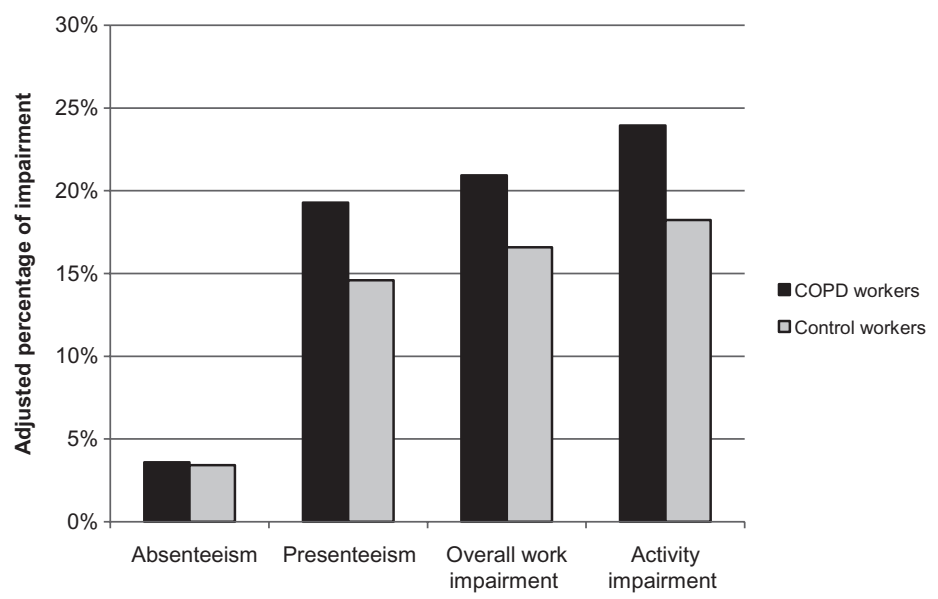

Figure 3 Mean levels of work productivity and activity impairment after adjusting for demographics and patient characteristics (comparing workers with COPD versus those without).

Note: Differences in presenteeism, overall work impairment, and activity impairment were found to be statistically significant $(P<0.000 \mathrm{I})$

Abbreviation: COPD, chronic obstructive pulmonary disease. 


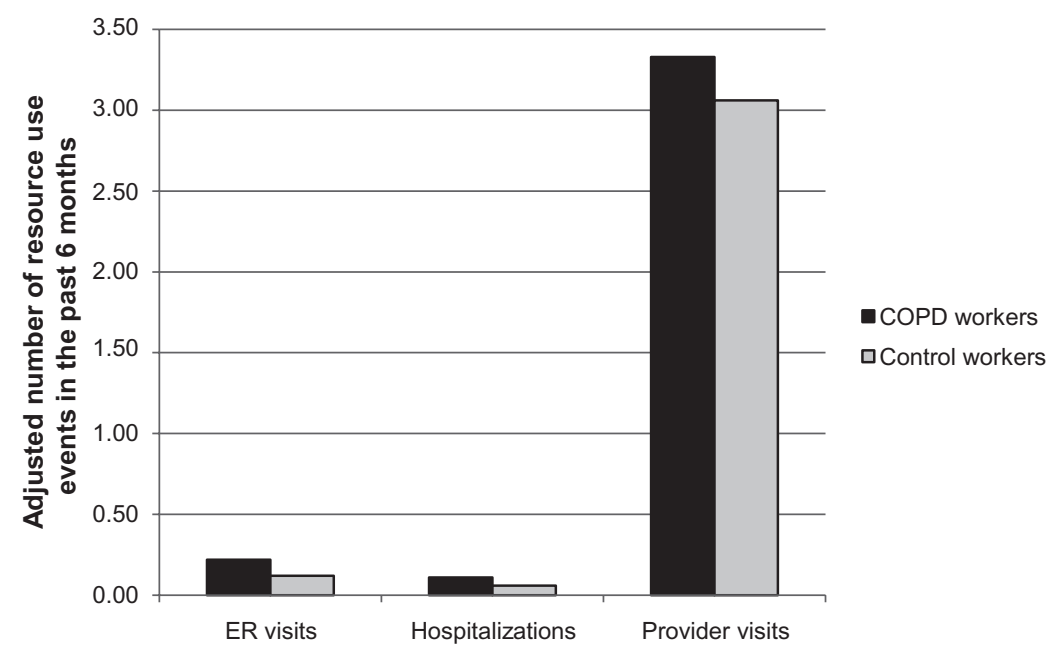

Figure 4 Mean levels of resource use after adjusting for demographics and patient characteristics (comparing workers with COPD versus those without). Note: Differences in ER visits, hospitalizations, and provider visits were found to be statistically significant $(P<0.000 \mathrm{I})$.

Abbreviations: COPD, chronic obstructive pulmonary disease; ER, emergency room.

has often grouped older and younger workers together, ${ }^{4}$ so that limiting the analyses to those aged 40-64 years helps homogenize the sample and isolates those in their peak earning years. This focus is also helpful in that most HRQoL studies have ignored those under the age of 65 years with COPD. ${ }^{4-7,19,20}$

The results of the current study suggest that workers diagnosed with COPD have significantly lower levels of HRQoL, even after controlling for health history and comorbidities. It is important to note that previous studies have often compared COPD workers to population norms (mean $=50$, standard deviation $=10$ ), without direct comparisons with control cohorts. ${ }^{5,6}$ Using such a direct comparison further emphasizes the impact that COPD can have on humanistic outcomes.

Previous studies have found COPD to be associated with reductions in workforce participation ${ }^{9,21}$ and increases in absenteeism. ${ }^{11}$ Although a trend toward greater absenteeism was observed here, it was not significant. However, significant differences between the COPD group and the control group were observed on presenteeism, a finding not previously reported. This result is important to emphasize because the effects of COPD may go unnoticed by employers who may focus on work absences rather than lost productivity while at work. Additionally, overall work impairment and activity impairment were also significantly higher among those diagnosed with COPD.

Lastly, health care resource utilization was significantly associated with COPD diagnosis. After accounting for demographic and health history variables, COPD workers were more likely to visit a traditional and non-traditional health care provider, but COPD workers did not report a higher number of traditional provider visits. COPD workers did, however, report significantly more ER visits and hospitalizations relative to controls. It is possible that the lack of additional provider visits may account for some of the humanistic and productivity loss detriments. Perhaps a lack of active management was a reason for poor outcomes and may explain higher rates of ER visits and hospitalizations. Future research is clearly necessary to examine these hypotheses.

\section{Limitations}

Because the NHWS is a cross-sectional survey, all relationships can only be expressed as associations and not causal relationships. Further research employing prospective and longitudinal designs may be useful in corroborating the burden of COPD as observed here, particularly as it relates to resource use. It is also important to note, though every attempt was made to rule out alternative explanations, that unmeasured variables may explain the relationships between group membership and health outcomes. Because of the selfreported nature, recall bias may have introduced additional error into the observed associations. Some patients may have incorrectly reported a COPD diagnosis when they do not have COPD and some patients may have failed to report a COPD diagnosis when they have COPD. Finally, although the NHWS is demographically representative of the US population because of the random stratified sampling framework, the final sample may differ in meaningful ways other than demographics, which could affect the size and direction of the relationships observed here. In other words, those who chose to respond to the survey may differ from those who chose not to respond (eg, health status, health care attitudes, etc) and 
these factors could artificially inflate or deflate the differences between groups.

\section{Conclusion}

Workers diagnosed with COPD reported significantly lower levels of HRQoL and higher levels of presenteeism, overall work impairment, activity impairment, ER visits, and hospitalizations. These effects remained even after adjusting for differences between the COPD group and control group in terms of comorbidities and other health history variables. Though absenteeism rates were similar between the two groups, employers should be aware that productivity losses were significantly higher among COPD workers as were costly ER visits and hospitalizations. Effective programs and policies may be necessary to better manage COPD workers in the workforce.

\section{Disclosure}

The NHWS is conducted by Kantar Health. Pfizer, Inc funded the analysis and preparation of this manuscript. MDD and JSW were both employed by Kantar Health; RPR, MM, and KHZ were employed by Pfizer, Inc; JS and HS were employed by Boehringer Ingelheim Pharmaceuticals, Inc, at the time of this study.

\section{References}

1. Punturieri A, Croxton TL, Weinman GG, Kiley JP. Chronic obstructive pulmonary disease: a view from the NHLBI. Am J Respir Crit Care Med. 2008;178(5):441-443.

2. Jones PW, Agusti AG. Outcomes and markers in the assessment of chronic obstructive pulmonary disease. Eur Respir J. 2006;27(4):822-832.

3. Minkoff NB. Analysis of the current care model of the COPD patient: a health outcomes assessment and economic evaluation. J Manag Care Pharm. 2005;11(6 Suppl A):S3-S7; quiz S20-S22.

4. Ketelaars CA, Schlosser MA, Mostert R, Abu-Saad HH, Halfens RJ, Wouters EF. Determinants of health-related quality of life in patients with chronic obstructive pulmonary disease. Thorax. 1996;51(1):39-43.

5. Stahl E, Lindberg A, Jansson SA, et al. Health-related quality of life is related to COPD disease severity. Health Qual Life Outcomes. 2005;3:56.

6. Garrido PC, Diez JD, Guiterrez JR, et al. Negative impact of chronic obstructive pulmonary disease on the health-related quality of life of patients. Results of the EPIDEPOC study. Health Qual Life Outcomes. $2006 ; 4: 31$
7. Garrido PC, Diez JD, Guiterrez JR, et al. Characteristics of chronic obstructive pulmonary disease in Spain from a gender perspective. BMC Pulm Med. 2009;9:2.

8. Yeo J, Karimova G, Bansal S. Co-morbidity in older patients with COPD - its impact on health service utilisation and quality of life, a community study. Age Ageing. 2006;35(1):33-37.

9. Sin DD, Stafinski T, Ng YC, Bell NR, Jacobs P. The impact of chronic obstructive pulmonary disease on work loss in the United States. Am J Respir Crit Care Med. 2002;165(5):704-707.

10. Darkow T, Kadlubek PJ, Shah H, Phillips AL, Marton JP. A retrospective analysis of disability and its related costs among employees with chronic obstructive pulmonary disease. J Occup Environ Med. 2007;49(1):22-30.

11. Halpern MT, Polzin J, Higashi MK, Bakst A. The workplace impact of acute exacerbations of chronic bronchitis (AECB); A literature review. COPD. 2004;1(2):249-254.

12. Darkow T, Chastek BJ, Shah H, Phillips AL. Health care costs among individuals with chronic obstructive pulmonary disease within several large, multi-state employers. J Occup Environ Med. 2008 50(10):1130-1138.

13. Bolge SC, Doan JF, Kannan H, Baran RW. Association of insomnia with quality of life, work productivity, and activity impairment. Qual Life Res. 2009;18(4):415-422.

14. Ware J Jr, Kosinski M, Turner-Bowker DM, et al. How to score version 2 of the SF-12v2 health survey (with a supplement documenting version 1). Lincoln, RI: QualityMetric, Inc; 2002.

15. Reilly MC, Zbrozek AS, Dukes EM. The validity and reproducibility of a work productivity and activity impairment instrument. Pharmacoeconomics. 1993;4(5):353-365.

16. Andreasson E, Svensson K, Berggren F. Validity of the work productivity and activity impairment questionnaire for patients with asthma (WPAI-asthma): Results from a web-based study. Presented at the ISPOR 6th Annual European Congress; November 9-11, 2003: Barcelona, Spain; 2003.

17. Dean BB, Crawley JA, Schmitt CM, Wong J, Ofman JJ. The burden of illness of gastro-oesophageal reflux disease: impact on work productivity. Aliment Pharmacol Ther. 2003;17(10):1309-1317.

18. Wahlqvist P, Karlsson M, Johnson D, Carlsson J, Bolge SC, Wallander MA Relationship between symptom load of gastro-oesophageal reflux disease and health-related quality of life, work productivity, resource utilization and concomitant diseases: survey of a US cohort. Aliment Pharmacol Ther. 2008;27(10):960-970.

19. Renwick DS, Connolly MJ. Impact of obstructive airways disease on quality of life in older adults. Thorax. 1996;51(5):520-525.

20. Sanchez FF, Faganello MM, Tanni SE, Lucheta PA, Padovani CR, Godoy I. Relationship between disease severity and quality of life in patients with chronic obstructive pulmonary disease. Braz J Med Biol Res. 2008;41(10):860-865.

21. Mannino DM, Braman S. The epidemiology and economics of chronic obstructive pulmonary disease. Proc Am Thorac Soc. 2007;4(7): $502-506$.
International Journal of COPD

\section{Publish your work in this journal}

The International Journal of COPD is an international, peer-reviewed journal of therapeutics and pharmacology focusing on concise rapid reporting of clinical studies and reviews in COPD. Special focus is given to the pathophysiological processes underlying the disease, intervention programs, patient focused education, and self management protocols.

\section{Dovepress}

This journal is indexed on PubMed Central, MedLine and CAS. The manuscript management system is completely online and includes a very quick and fair peer-review system, which is all easy to use. Visit http://www.dovepress.com/testimonials.php to read real quotes from published authors. 